\title{
LA-UR-14-23304
}

Approved for public release; distribution is unlimited.

Title: $\quad$ IMPACT Project Integrated Modeling of Perturbations in Atmospheres for Conjunction Tracking A New Orbital Prediction Model to Avoid Collisions in Space

Author(s): $\quad$ Godinez Vazquez, Humberto C.

Intended for: $\quad$ report slides for LANL Institutional Computing program

Issued: $\quad$ 2014-05-09

Disclaimer:

Los Alamos National Laboratory, an affirmative action/equal opportunity employer,is operated by the Los Alamos National

Security, LLC for the National NuclearSecurity Administration of the U.S. Department of Energy under contract DE-AC52-06NA25396.

By approving this article, the publisher recognizes that the U.S. Government retains nonexclusive, royalty-free license to publish or reproduce the published form of this contribution, or to allow others to do so, for U.S. Government purposes.

Los Alamos National Laboratory requests that the publisher identify this article as work performed under the auspices of the

U.S. Departmentof Energy. Los Alamos National Laboratory strongly supports academic freedom and a researcher's right to publish; as an institution, however, the Laboratory does not endorse the viewpoint of a publication or guarantee its technical correctness. 


\section{IMPACT Project}

Integrated Modeling of Perturbations in Atmospheres for Conjunction Tracking

A New Orbital Prediction Model to Avoid Collisions in Space

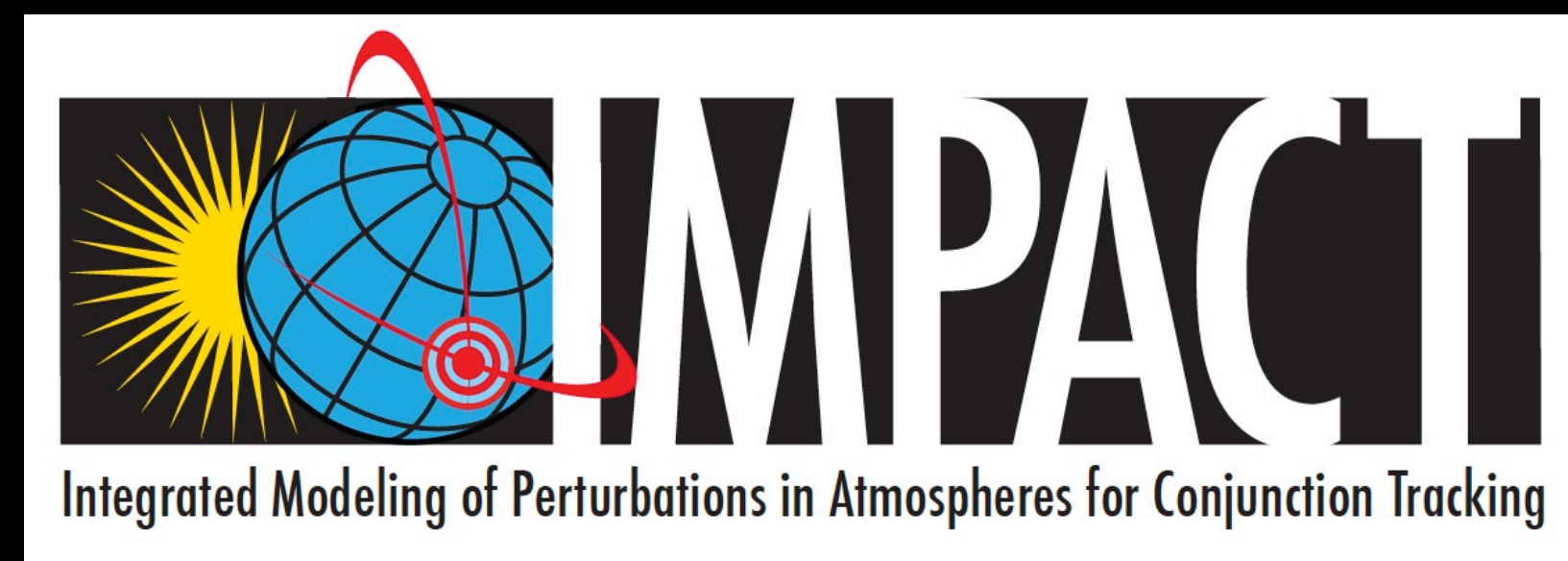

\section{PI: Humberto C. Godinez, T-5 hgodinez@lanl.gov}




\section{Integrated Modeling of Perturbations in Atmospheres for Conjunction Tracking}

- We are developing a new physics-based satellite drag model to accurately predict satellite paths and to prevent collisions in space.

- Institutional Computing resources have been used to

- develop a data assimilative thermospheric density model

- employ Direct Simulation Monte Carlo calculations of the satellite drag coefficient

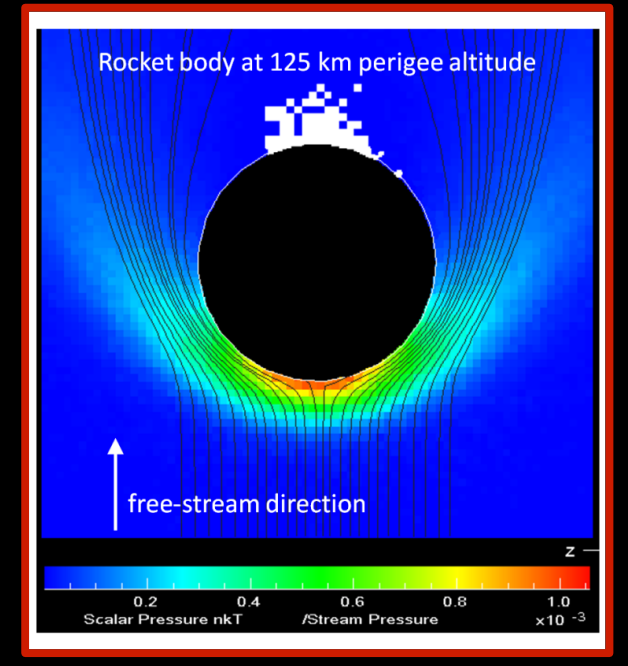

Rocket body simulation in low Earth orbit

- First results include

- Ensemble Kalman Filter method used to estimate the coupling between solar drivers and thermospheric density changes

- First self-consistent drag coefficients calculations using the physicsbased and assimilative thermosphere model 


\section{Assimilative thermosphere model is filling gaps in sparse data set}

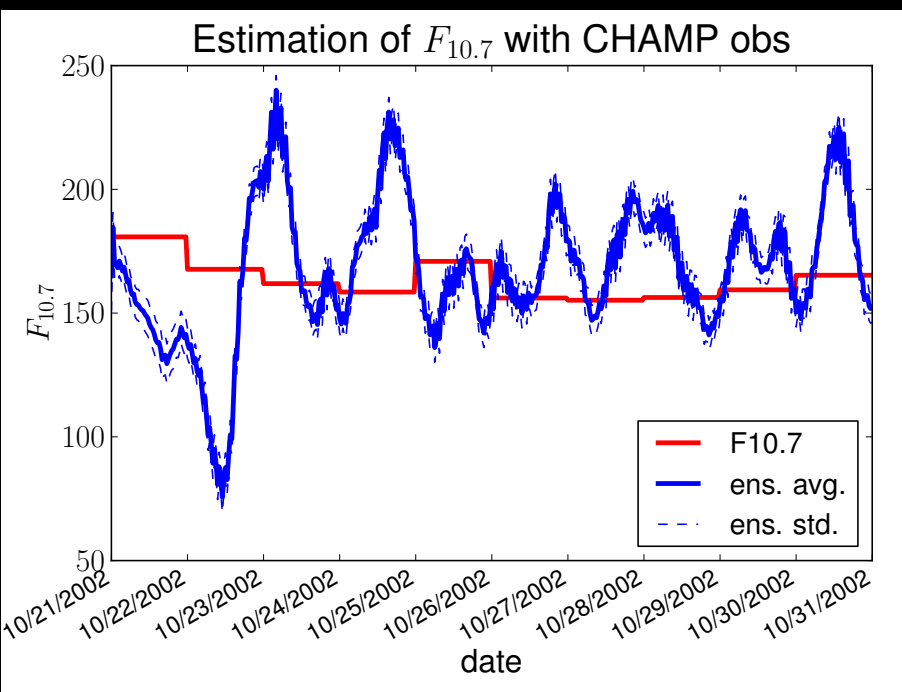

Data Assimilation: Enhancing the GITM model using a localized ensemble transform Kalman Filter. Top left: Estimated F10.7 using assimilation to drive GITM. Top right: GITM result after assimilating CHAMP data. Bottom right: Multi-model simulation (GITM, TIE-GCM, MSIS) compared to CHAMP data and multi-model average.
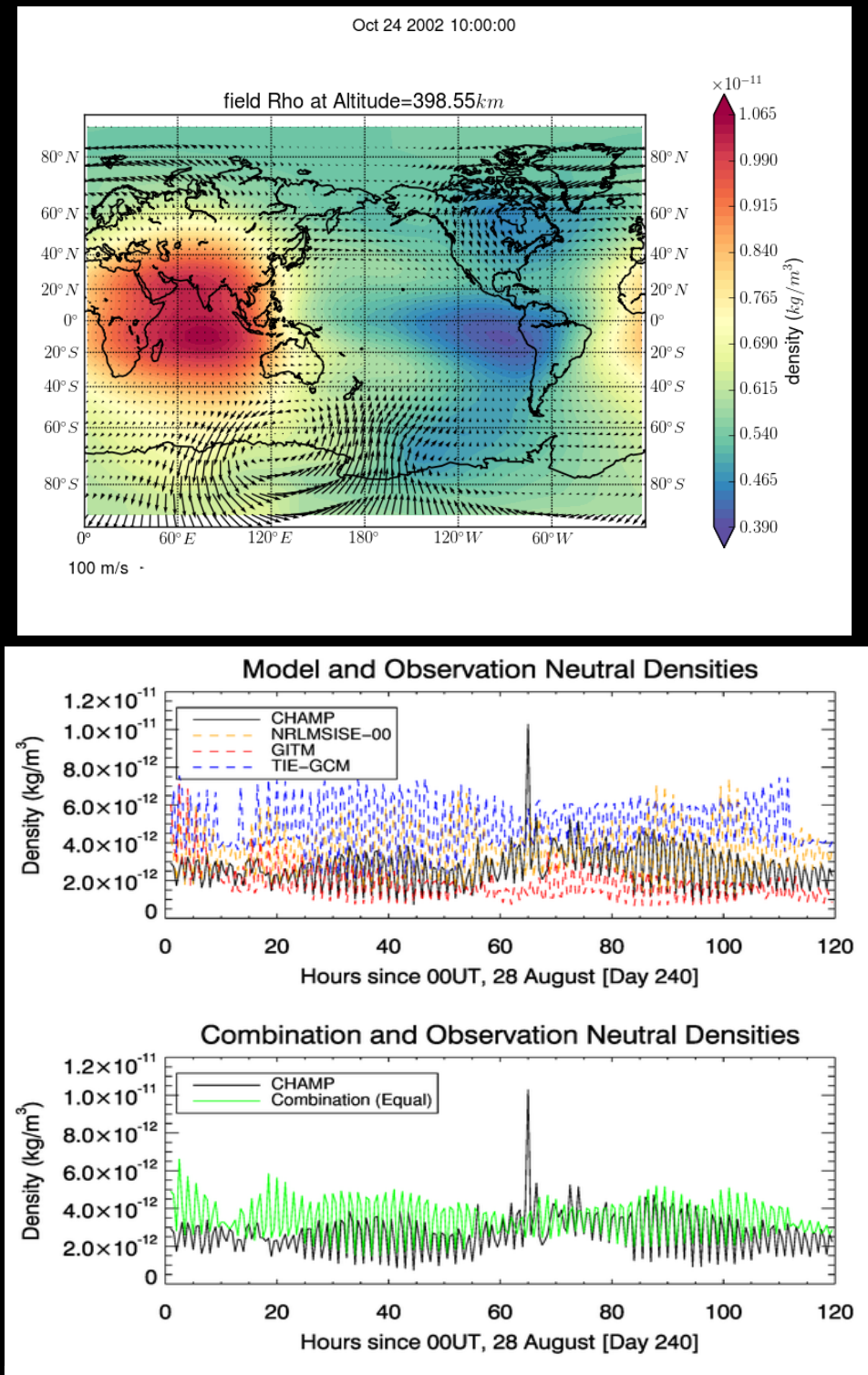


\section{Drag coefficient calculation with Direct Simulation Monte Carlo Method (DSMC)}

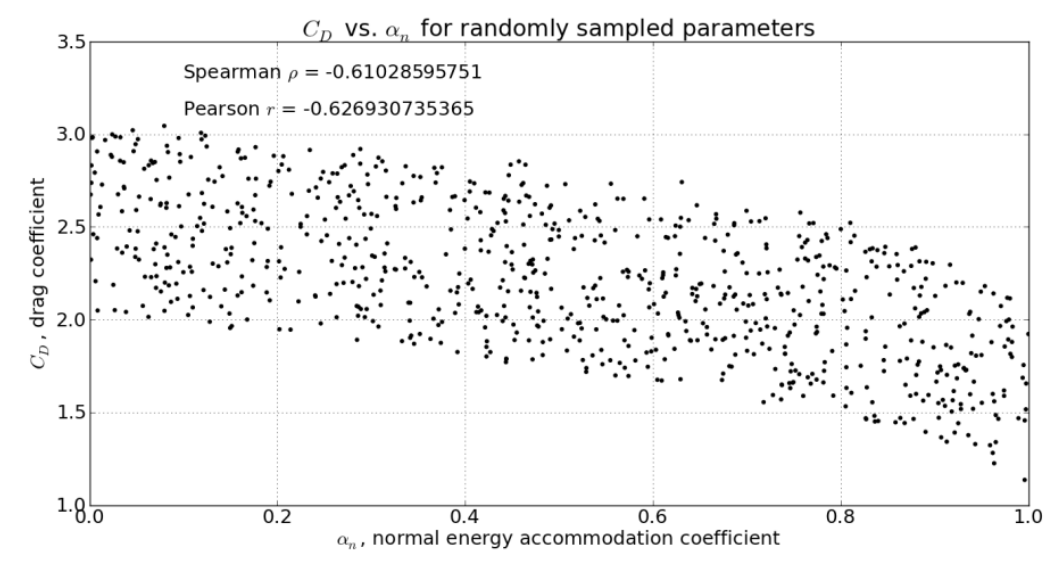

Drag Coefficient Modeling: The Drag coefficient is a function of chemical composition, thermospheric temperature, thermospheric densities, etc. All of these are affected by space weather. We are the first to perform self-consistent calculations of satellite drag coefficients using our physics-based model of neutral densities and chemical composition of the upper atmosphere. Top plot: 1000 simulated drag coefficients as a function of energy accommodation coefficient. Bottom plot: the associated error compared to a modified closed-form solution. Each data point represents a full serial Direct Simulation Monte Carlo (DSMC) simulation for the drag coefficient of a sphere. The input parameters for each simulation are sampled from the global parameter space using Latin Hypercube Sampling. 